\title{
Incidence and Risk Factors of the Intraoperative Localization Failure of Nonpalpable Breast Lesions by Radio-Guided Occult Lesion Localization: A Retrospective Analysis of 579 Cases: Reply
}

\author{
Sergio Bernardi · Serena Bertozzi • \\ Ambrogio P. Londero
}

Published online: 8 November 2012

(C) Société Internationale de Chirurgie 2012

We are pleased about your interest in our article that focused on the difficulty of detecting nonpalpable breast lesions [1, 2]. The failure of intraoperative detection of these lesions by radio-guided lesion localization (ROLL) or by wire hook is an important topic because they represent the majority of breast lesions.

It should be mandatory that intraoperative radiological examination be performed on every surgical specimen obtained by breast-conserving surgery, whether obtained by mammography (in the most cases) or by ultrasonography in cases of negative preoperative mammography. In fact, radiological evaluation of surgical resection margins, especially in the absence of subsequent, intraoperative frozen section examination, could ensure correct targeting of the lesion and could identify the size of the margins needed to remove all of the tumor.

We agree that injecting contrast medium together with the radiotracer can be helpful for the radiologist. It probably improves both preoperative targeting and intraoperative radiologic evaluation of the specimen. It also probably improves the accuracy of preoperative and intraoperative imaging comparison [1].

The experience of the multidisciplinary team involved in the ROLL procedure is the main factor influencing its

\footnotetext{
S. Bernardi · S. Bertozzi $(\bowtie)$

Department of Surgery, AOU "Santa Maria della Misericordia", Piazzale SM della Misericordia, 15, 33100 Udine, Italy e-mail: dr.bertozzi@gmail.com; sere_d.estate@libero.it

S. Bernardi

Unit of Surgery, OC Latisana, 33053 Latisana, UD, Italy

A. P. Londero

Clinic of Obstetrics and Gynecology, AOU "Santa Maria della

Misericordia", 33100 Udine, Italy
}

success $[1,2]$. As nonpalpable breast lesions are common in our country, we perform ROLL-guided surgery in small surgical units. In our opinion, however, it is advisable that the radio-guided radiotracer be injected by an experienced team at a center with a high workload and an experienced, dedicated radiology team. After correctly targeting the lesion, the surgical excision could then be performed in a smaller center that is within a reasonable distance from the center with the experienced team.

Finally, we would be pleased to read prospective results published by colleagues and compare our data with theirs. We could then perhaps find a solution for reducing the number of times the ROLL procedure fails to identify nonpalpable breast lesions.

\section{References}

1. Postma EL, Verkooijen HM, van den Bosch MAAJ et al (2012) Incidence and risk factors of the intraoperative localization failure of nonpalpable breast lesions by radio-guided occult lesion localization: a retrospective analysis of 579 cases-reply. World J Surg. doi:10.1007/s00268-012-1807-6

2. Bernardi S, Bertozzi S, Londero AP et al (2012) Incidence and risk factors of the intraoperative localization failure of nonpalpable breast lesions by radio-guided occult lesion localization: a retrospective analysis of 579 cases. World J Surg 36:1915-1921. doi:10.1007/s00268-012-1577-1 